\title{
Patient-Specific Mitral Leaflet Segmentation from 4D Ultrasound
}

\author{
Robert J. Schneider ${ }^{1}$, Neil A. Tenenholtz ${ }^{1}$, Douglas P. Perrin ${ }^{1,2}$, \\ Gerald R. Marx ${ }^{3}$, Pedro J. del Nido ${ }^{2}$, and Robert D. Howe ${ }^{1}$ \\ 1 Harvard School of Engineering and Applied Sciences, Cambridge, MA, USA \\ ${ }^{2}$ Department of Cardiac Surgery, Children's Hospital, Boston, MA, USA \\ ${ }^{3}$ Department of Cardiology, Children's Hospital, Boston, MA, USA
}

\begin{abstract}
Segmenting the mitral valve during closure and throughout a cardiac cycle from four dimensional ultrasound (4DUS) is important for creation and validation of mechanical models and for improved visualization and understanding of mitral valve behavior. Current methods of segmenting the valve from 4DUS either require extensive user interaction and initialization, do not maintain the valve geometry across a cardiac cycle, or are incapable of producing a detailed coaptation line and surface. We present a method of segmenting the mitral valve annulus and leaflets from 4DUS such that a detailed, patient-specific annulus and leaflets are tracked throughout mitral valve closure, resulting in a detailed coaptation region. The method requires only the selection of two frames from a sequence indicating the start and end of valve closure and a single point near a closed valve. The annulus and leaflets are first found through direct segmentation in the appropriate frames and then by tracking the known geometry to the remaining frames. We compared the automatically segmented meshes to expert manual tracings for both a normal and diseased mitral valve, and found an average difference of $0.59 \pm 0.49 \mathrm{~mm}$, which is on the order of the spatial resolution of the ultrasound volumes $(0.5-1.0 \mathrm{~mm} /$ voxel $)$.
\end{abstract}

\section{Introduction}

The mitral valve is a thin leaflet structure responsible for maintaining unidirectional blood flow from the left atrium to the left ventricle. Mitral valve disease, however, is one of the most prevalent among valve diseases [1], often necessitating surgical intervention. Despite this, gathering detailed information about valve dynamics on a patient-specific basis is rarely performed due to limited methods to extract valve geometry.

To better understand the mitral valve, several studies have attempted to simulate valve closure with either generic or patient-specific knowledge of the valve geometry 23]. Those that have used patient-specific geometry have generally done so by generating models from three-dimensional ultrasound (3DUS) [3]. The advantages of generating models from ultrasound are that ultrasound is an imaging modality that is non-ionizing and inexpensive, and is commonly used clinically to image the valve for diagnostic and interventional purposes.

G. Fichtinger, A. Martel, and T. Peters (Eds.): MICCAI 2011, Part III, LNCS 6893, pp. 520-527, 2011.

(C) Springer-Verlag Berlin Heidelberg 2011 
The challenge with model generation from 3DUS is that a limited number of methods exist that are able to accurately and robustly segment a patient-specific $3 \mathrm{D}$ valve and its components with minimal input and interaction required by the user. A volumetric segmentation of the leaflets using an intensity-based level set method was shown in [4, but the segmentation method was not able to isolate the valve from the surrounding tissue and could not separate anterior from posterior leaflets upon coaptation. The work by Burlina et al., 2009 uses a level set method and thin tissue detector to define the valve geometry and surroundings, but requires extensive user interaction and relies on the assumption of a planar annulus 3. Furthermore, the segmentation is performed in only a single frame showing an open valve. The closed configuration of the valve is estimated by modeling the mechanics of the valve, but the accuracy of this approach is not validated. The work by Ionasec et al., 2010 described a sophisticated system that fits a mitral and aortic model to the respective valve using machine learning techniques and a large database of manually delineated points [5]. In the context of mitral valve segmentation, however, the mitral valve model lacked fine leaflet detail due to the model being fit to only a few locations. Additionally, the model appeared to only roughly estimate the coaptation line and did not appear to be able to generate an estimate of coaptation length, which is a measure commonly used by clinicians to assess valve competency.

To address these issues, we present a 4D mitral valve segmentation method that can generate a detailed 4D patient-specific annulus and leaflet geometry. As most modeling efforts and clinical interest revolves around the closing of the valve, the method is designed to capture the valve geometry during this phase of the cardiac cycle. However, the method could easily be altered to find the leaflet geometry throughout an entire 4D ultrasound (4DUS) sequence. The method requires only the selection of two frames from an ultrasound sequence - one before the valve starts to close and another after the valve closes - and the selection of a point near the center of the closed valve. The method then automatically finds the annulus and leaflets during valve closure. By taking into account valve behavior and handling leaflet collisions, a detailed coaptation line and surface are generated. It is important to make the distinction that we are not simulating the mechanics of the valve, but rather are presenting a method to delineate the valve as seen in a 4DUS sequence. The extracted geometry is intended for inputs to mechanical models or surgical simulators, improved visualization, diagnostics, or for inverse modeling purposes.

\section{Methods and Materials}

\subsection{Constructing Geometric Priors}

The mitral valve segmentation method operates by first constructing a geometric prior of the valve which is then propagated to all other frames of interest. The valve geometry is constructed by first finding the annulus, which is then used to enforce constraints about where to find the leaflets. To find the annulus, the user-selected frame showing a closed mitral valve and the selected valve center 


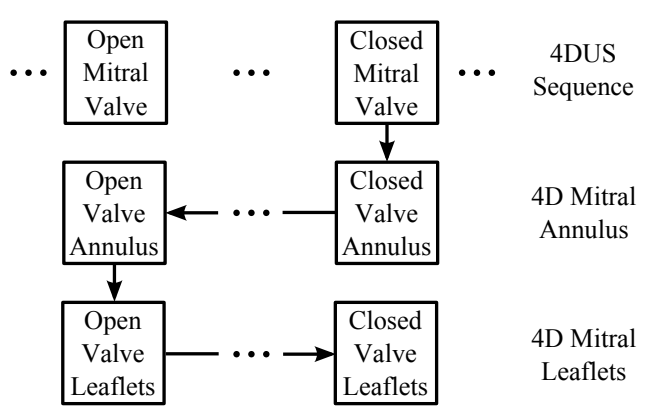

Fig. 1. Summary of 4DUS segmentation of the mitral valve. A user manually specifies the frames at the start and end of valve closure, and also a point near the center of the closed mitral valve. The annulus is found through segmentation and then tracking, starting from the closed valve and proceeding to the open. The leaflets are then found through segmentation and then tracking, starting from the open valve and proceeding to the closed.

point are used in a direct annulus segmentation method [6]. The segmented annulus is then tracked to the remaining frames of interest using a variation of the Lucas \& Kanade optical flow algorithm [7, similar to that shown in 8]. The annulus in the frame selected by the user as showing the mitral valve in the open configuration before the valve begins to close is then used in a direct leaflet segmentation method to generate a detailed patient-specific triangular mesh at the leaflets [9]. The following sections describe how the mesh is evolved during valve closure such that the geometry mirrors that of the valve in the 4DUS images.

\subsection{Evolving the Leaflet Mesh}

The mitral valve mesh is evolved frame to frame by treating the mesh as an active surface [10, where the nodes of the mesh at the annulus are fixed according to the annulus segmentation and tracking results, and the rest of the mesh is free to evolve. The active surface is encouraged to track the top (atrial) surface of the leaflets as seen in the 3DUS images, regulated using internal edge and bending forces, and designed to handle collisions between leaflets. The active surface is then evolved according to the force equation

$$
\mathbf{F}_{\text {valve }}=w_{\text {image }} \mathbf{F}_{\text {image }}+w_{\text {edge }} \mathbf{F}_{\text {edge }}+w_{\text {bend }} \mathbf{F}_{\text {bend }}+w_{\text {collision }} \mathbf{F}_{\text {collision }}
$$

where $w_{\text {image }}, w_{\text {edge }}, w_{\text {bend }}$, and $w_{\text {collision }}$ are scalar weights. The nodes, $\mathbf{x}$, of the mesh (except for annulus nodes) are then evolved such that $\mathbf{x}^{k+1}=\mathbf{x}^{k}+d t \mathbf{F}_{\text {valve }}$, where $d t$ is a time step. The annulus nodes, $\mathbf{x}_{\text {annulus }}$, are evolved according to the displacement, $\mathbf{D}_{\text {annulus }}$, and correspondence determined from tracking the annulus from frame to frame. Displacing the annulus nodes is carried out over $k_{\text {annulus }}$ time steps, such that $\mathbf{x}_{\text {annulus }}^{k+1}=\mathbf{x}_{\text {annulus }}^{k}+\delta_{\text {annulus }}\left(\frac{1}{k_{\text {annulus }}}\right) \mathbf{D}_{\text {annulus }}$, where $\delta_{\text {annulus }}=1$ when $k<k_{\text {annulus }}$ and zero otherwise. 
Image Force. The image force drives the mesh so that it tracks the top surface of the leaflets as seen in the 3DUS volumes. The top surface is tracked as this is the surface that defines leaflet coaptation. One component of the image force is derived from the image gradient of a Gaussian convolved thin tissue detector image [6], $\nabla \mathrm{G}_{\sigma}$ (TTD). This component drives the valve mesh toward the center of the leaflets and helps ensure that leaflets are driven to roughly the desired location even in the presence of large displacements. We encourage the mesh to find the atrial surface of the leaflets using the gradient of the target frame's ultrasound intensity, $\mathbf{I}_{\text {target }}$, along the node normal, N. Having already defined the normal at each node to point toward the atrial side of the leaflets, the atrial surface of the leaflets should be at a location where $\frac{\partial \mathbf{I}_{\text {target }}}{\partial \mathbf{N}}$ is at a minimum (Figure 2). The image force is then specified as

$$
\mathbf{F}_{\text {image }}=\nabla \mathrm{G}_{\sigma}(\mathbf{T T D}) \cdot \mathbf{N}-\delta_{\text {image }} \frac{\partial^{2} \mathbf{I}_{\text {target }}}{\partial \mathbf{N}^{2}}
$$

where $\delta_{\text {image }}=1$ when a node is at a location where $\frac{\partial \mathbf{I}_{\text {target }}}{\partial \mathbf{N}}<0$ and zero otherwise. This is to prevent a node that is below the leaflet from being pushed even further below the leaflets.
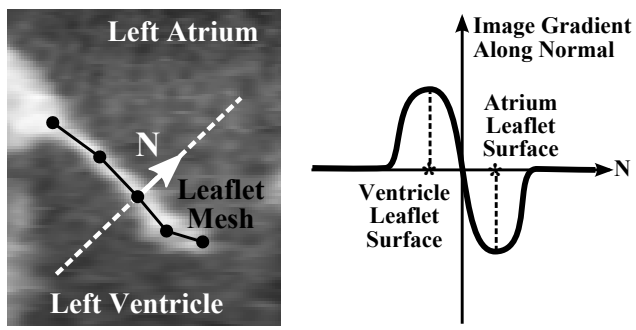

Fig. 2. Simplified depiction of the image gradient along the node normal. (Left) Ultrasound volume shown with the position of a mesh and the direction of a node normal. (Right) A simplified graph showing the gradient of ultrasound intensity along the normal. To capture the atrial side of the leaflets, nodes are forced in a direction so as to minimize the value of the gradient.

Edge Force and Artificial Strain. We maintain the geometric prior as determined from the initial leaflet segmentation by regulating the edge lengths of the mesh. We do this by imparting the edge force

$$
\mathbf{F}_{\text {edge }}=\sum_{e=1}^{E} \delta_{\text {edge }}\left(L_{e}-L_{e, o}\right)
$$

where $\delta_{e d g e}=\delta_{c}$ when $L_{e}-L_{e, o}<0$ (i.e. edge is in compression) and $\delta_{e d g e}=\delta_{t}$ when $L_{e}-L_{e, o}>0$ (i.e. edge is in tension). The original edge lengths are represented by $L_{e, o}$ and the number of edges attached to each node by $E$. For our design, we make $\delta_{c}<\delta_{t}$ as a way of penalizing longer edges over shorter. 
A key issue in creating segmented models of the mitral valve is identifying the regions where the leaflets overlap when closed, as this determines the propensity for the valve to leak. This is challenging because it is impossible to discern separate leaflets in this coaptation region once the valve is closed. This necessitates tracking the leaflets as the valve closes, and approximating the stretch that ensues as the leaflets are pressurized. Studies on the mechanical properties of the valve have shown that leaflets stretch by roughly $35 \%$ in the radial direction and $20 \%$ in the circumferential direction [1] due to the pressure gradient across the valve, and because the leaflet is constrained at the annulus and by chords. As accurate chordal structure is extremely difficult to obtain from 3DUS, leaflet strain is applied artificially throughout the closure of the valve. Having determined the radial and circumferential components of each edge when the leaflet mesh is first segmented, the reference edge lengths, $L_{e, o}$ are adjusted accordingly throughout the valve. Making $\delta_{c}<\delta_{t}$ makes this artificial strain not a hard constraint but a soft constraint, and making $\delta_{t}$ large ensures that the leaflets do not stretch much more than the applied artificial strain. In our design, the applied strain rate is such that the leaflets are fully strained by halfway through leaflet closure to ensure that leaflets have the opportunity to coapt.

Bending Force. Due to the noise in 3DUS images, the image force has the potential to displace the leaflet mesh in ways uncharacteristic of an actual valve and in ways which cannot be corrected by the edge force. We therefore impart a bending force which tends to straighten the leaflets to further regularize the surface geometry.

$$
\mathbf{F}_{\text {bend }}=\arccos \left(\mathbf{N}_{i} \cdot \mathbf{N}_{j}\right)
$$

The bending force is computed at each edge which is shared by two triangular faces whose respective normals are $\mathbf{N}_{i}$ and $\mathbf{N}_{j}$. The force is then imparted appropriately to the edge nodes and the nodes opposite the edge from each face.

Collision Force. To resolve collisions between leaflets and ultimately allow for the formation of a detailed coaptation surface, a collision force is computed between edges that are close to colliding, where potentially colliding edges are determined from a grid-based culling operation. If the distance between two edges is $d_{c}$, the force between the edges is $f_{c}=\exp \left(d_{c}\right)$. The collision force at each node is then found as

$$
\mathbf{F}_{\text {collision }}=\sum_{e=1}^{E} f_{c, e}
$$

where $E$ again represents the number of edges attached to a node.

Tether Force. As previously mentioned, segmenting the chordal structure of the mitral valve from 3DUS is extremely difficult. The chords act to constrain the free edge of the leaflets. Without chords, the free edge of the leaflets has the potential to flail uncontrollably. The same is true for the leaflet mesh in the presence of the image force. We therefore found it necessary to impart a 
comparatively small tethering force to the free edge of the leaflets. This is done by redirecting the image force at the free edge by adding a component to the normal that points toward the left ventricle. If the magnitude of the image force is $f_{\text {image,o }}$, which is directed along node normal $\mathbf{N}$, then the new image force at the free edge is

$$
\mathbf{F}_{\text {image }}=\left(\frac{f_{\text {image }, o}}{2}\right) \frac{\mathbf{N}+\mathbf{A}_{\text {valve }}}{\left|\mathbf{N}+\mathbf{A}_{\text {valve }}\right|}
$$

where $\mathbf{A}_{\text {valve }}$ is a vector pointing along the axis of the valve toward the left ventricle which is determined when the leaflet mesh is first segmented.

\section{Results}

We assessed the accuracy of the presented 4D mitral valve segmentation method using retrospective ECG-gated data acquired using a transesophageal approach (iE33 Echocardiography System with a transthoracic X7-2t probe, Philips Healthcare, Andover, MA, USA). Frames at the start and end of mitral valve closure and a point near the center of the closed mitral valve were manually selected. The valve weights $\left(w_{\text {image }}=0.05, w_{\text {bend }}=0.15, w_{\text {edge }}=0.02, w_{\text {collision }}=\right.$ $\left.2, \delta_{t}=5, \delta_{c}=1, d t=1\right)$ were tuned on a single data set to ensure stability and adequate tracking, and the method validated on two other data sets.

The results of the segmentation for the two data sets - one showing a normal mitral valve and another showing a stenotic mitral valve - at selected times during valve closure can be seen in Figure 3. To validate the segmentation method, the atrial side of the leaflets for the two data sets were manually traced by an expert in cut planes taken every $10^{\circ}$ about the valve axis. This was done in every frame during valve closure, amounting to over 50 frames and over 1000 cut planes between the two data sets. The average differences between the manual tracings and automatically segmented meshes for the first data set (normal mitral valve), second data set (diseased mitral valve), and across both data sets were $0.84+/-0.65 \mathrm{~mm}(\mathrm{RMS}=1.06 \mathrm{~mm}), 0.48+/-0.34 \mathrm{~mm}(\mathrm{RMS}=0.59 \mathrm{~mm})$, and $0.59+/-0.49 \mathrm{~mm}$ (RMS $=0.77 \mathrm{~mm})$, respectively. These differences were on the order of the spatial resolution of the ultrasound volumes, which were $0.5-1.0 \mathrm{~mm} /$ voxel. This analysis makes the presented algorithm one of the most thoroughly validated $4 \mathrm{D}$ mitral leaflet segmentation methods.

In showing the mesh location relative to the ultrasound images in Figure 3, it can be seen that the segmentation accurately finds the valve location throughout valve closure. Additionally, the $3 \mathrm{D}$ mesh shows that a detailed coaptation surface is delineated. The same parameters were used for both studies, with the exception that the artificial strains for the stenotic mitral valve were made to be half those of the normal (i.e. instead of using $35 \%$ strain in the radial direction and $20 \%$ in the circumferential, we used $17 \%$ and $10 \%$, respectively), which is an estimate for the mechanical properties of stenotic leaflet tissue. 

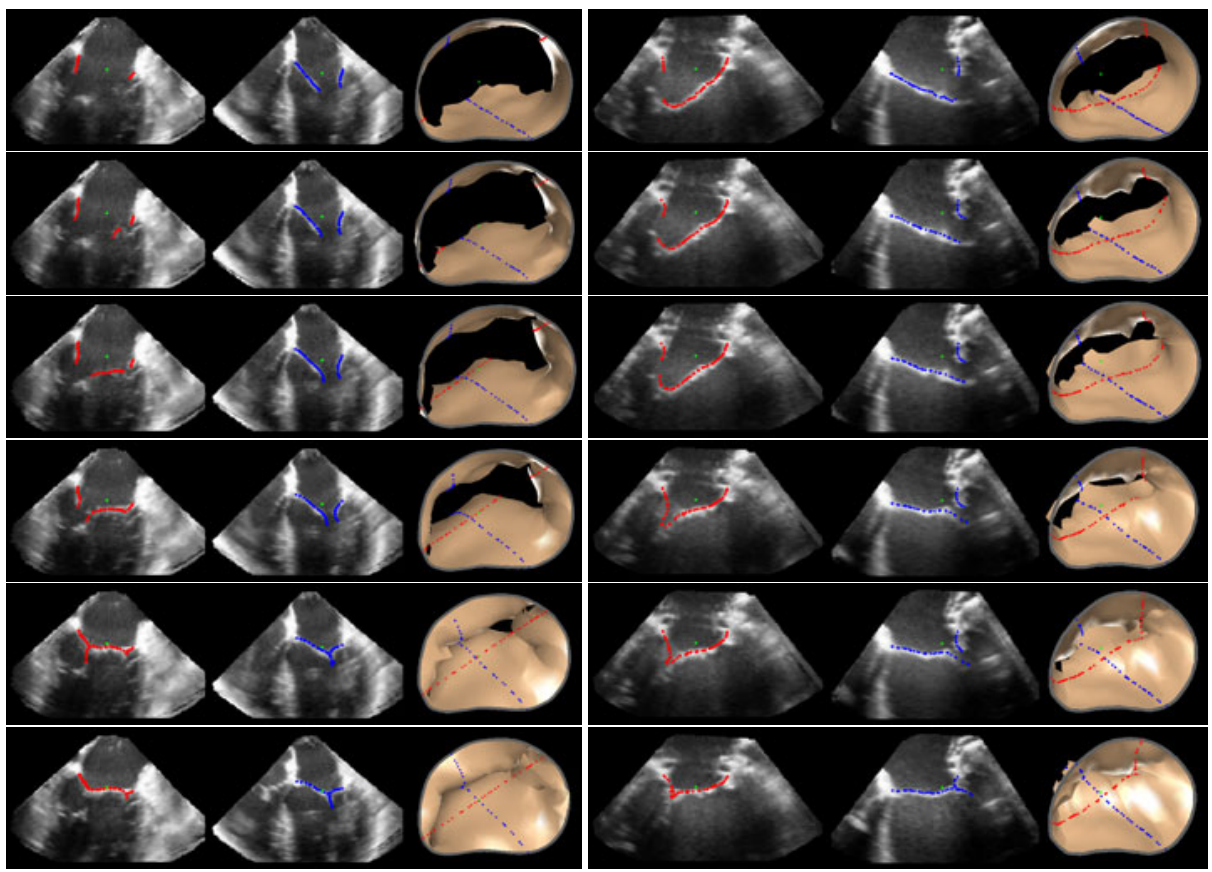

Fig. 3. Results of the mitral valve segmentation method during valve closure for a $\left(1^{\text {st }}\right.$ Column) normal and ( $2^{\text {nd }}$ Column) stenotic mitral valve. The comparison of the mesh location in the image planes (red and blue points) relative to the leaflets as seen in the images show the effectiveness of the $4 \mathrm{D}$ segmentation method for ultrasound.

\section{Discussion}

The presented mitral valve segmentation method is capable of finding the location of the mitral valve throughout valve closure, and could be modified to segment an entire cardiac cycle. The benefit of the presented method is that, with minimal user input, the method can segment the mitral valve in each frame during valve closure and is able to generate a detailed coaptation region. This is the first method capable of segmenting such patient-specific detail from a 4DUS sequence. The method benefits from assuming that a geometric prior can be very simply altered and displaced to track the desired anatomy throughout a give sequence. Were we not to assume a geometric prior and rather just segment the leaflets as seen in each separate image, the approach would be similar to that in [5], where a detailed coaptation region was not found.

As emphasized, the presented method is not performing mechanical modeling of the mitral valve. However, the method could be used in several aspects of modeling. The construction of the geometric priors could be used as a modeling input. Material properties could be determined by finding the strain values in the radial and circumferential directions that results in the best segmentation. Lastly, the 4D segmentations can be used to validate their predictions. As such, coupling segmentation and mechanical modeling could prove beneficial. 
The constructed geometric priors in this study did not include the mitral valve chords. These structures are too small to be accurately segmented from three-dimensional ultrasound. For this reason, several assumptions, such as artificial strain and tethering forces, had to be made in the segmentation method to account for this lack of information. Future efforts will include specializing ultrasound data acquisition for the collection of the desired chords.

Acknowledgments. This work was supported by the U.S. National Institutes of Health under grant NIH R01 HL073647-06.

\section{References}

1. Nkomo, V., Gardin, J., Skelton, T., Gottdiener, J., Scott, C.: Enriquez-Sarano, M.: Burden of valvular heart diseases: a population-based study. The Lancet 368, 1005-1011 (2006)

2. Hammer, P., Vasilyev, N., Perrin, D., del Nido, P., Howe, R.: Fast image-based model of mitral valve closure for surgical planning. In: MICCAI 2008 Workshop Proceedings: Computational Biomechanics for Medicine III, New York, NY, pp. 15-26 (2008)

3. Burlina, P., Sprouse, C., DeMenthon, D., Jorstad, A., Juang, R., Contijoch, F., Abraham, T., Yuh, D., McVeigh, E.: Patient-specific modeling and analysis of the mitral valve using 3d-tee. In: Navab, N., Jannin, P. (eds.) IPCAI 2010. LNCS, vol. 6135, pp. 135-146. Springer, Heidelberg (2010)

4. Shang, Y., Yang, X., Zhu, L., Deklerck, R., Nyssen, E.: Region competition based active contour for medical object extraction. Computerized Medical Imaging and Graphics 32, 109-117 (2008)

5. Ionasec, R., Voigt, I., Georgescu, B., Wang, Y., Houle, H., Vega-Higuera, F., Navab, N., Comaniciu, D.: Patient-Specific Modeling and Quantification of the Aortic and Mitral Valves From 4-D Cardiac CT and TEE. IEEE Transactions on Medical Imaging 29, 1636-1651 (2010)

6. Schneider, R., Perrin, D., Vasilyev, N., Marx, G., del Nido, P., Howe, R.: Mitral Annulus Segmentation from 3D Ultrasound Using Graph Cuts. IEEE Transactions on Medical Imaging 29, 1676-1687 (2010)

7. Lucas, B., Kanade, T.: An iterative image registration technique with an application to stereo vision. In: Proc. 7th International Joint Conference on Artificial Intelligence, Vancouver, B.C., Canada, pp. 674-679 (1981)

8. Veronesi, F., Corsi, C., Caiani, E., Sugeng, L., Weinert, L., Mor-Avi, V., Lang, R., Lamberti, C.: Semi-automatic tracking for mitral annulus dynamic analysis using real-time 3D echocardiography. Computers in Cardiology 33, 113-116 (2006)

9. Schneider, R., Burke, W., Marx, G., del Nido, P., Howe, R.: Modeling mitral valve leaflets from three-dimensional ultrasound. In: Metaxas, D., Axel, L. (eds.) FIMH 2011. LNCS, vol. 6666, pp. 215-222. Springer, Heidelberg (2011)

10. Terzopoulos, D., Witkin, A., Kass, M.: Constraints on deformable models: Recovering 3D shape and nonrigid motion. Artificial Intelligence 36, 91-123 (1988)

11. Grashow, J., Yoganathan, A., Sacks, M.: Biaixal stress-stretch behavior of the mitral valve anterior leaflet at physiologic strain rates. Annals of Biomedical Engineering 34, 315-325 (2006) 